\title{
Analysis of Target Tracking Algorithm in Thermal Imagery
}

\author{
Umesh Gupta \\ M.E. Student \\ Department of Computer \\ Science and Engineering \\ NITTTR, Chandigarh
}

\author{
MaitreyeeDutta, $\mathrm{PhD}$ \\ Associate Professor \\ Department of Computer \\ Science and Engineering \\ NITTTR, Chandigarh
}

\author{
Mahesh Vadhavaniya \\ Lecturer \\ Department of Instrumentation \\ and Control Engineering \\ Govt. Polytechnic, Gandhinagar
}

\begin{abstract}
Target tracking plays a vital role in the development of battlefield surveillance, airspace surveillance and Border Patrolling. The rapid uses of infrared imagery in target tracking prevents from a wide range of attacks in border security, sea shore security. Infrared imagery is an effective method to cluster heat generating targets and it can penetrate fog, haze, dust, smoke, snow, rain and extreme darkness operate at day and night. Infrared imagery is one of the major and efficient defensive medium in surveillance and monitoring activity. In this paper, an introduction of target tracking algorithms in infrared imagery is discussed and three detection algorithms such as single Reference Frame, Moving Average and Temporal Median Filter with tracking algorithm are implemented and analyzed on multiple targets dataset. This will open the new area for the researcher in the research field of security.
\end{abstract}

\section{Keywords}

Infrared Imagery, Target Tracking Algorithms, Target Detection Algorithms, Performance parameters.

\section{INTRODUCTION}

\subsection{Infrared Imagery}

A thermal imaging device gives the ability to see targets in darkness or smoke. Thermal imaging is a technology that creates a photographic image or video sequence of light emitted by an object at terrestrial temperatures. Infrared (IR) thermal imaging, also often briefly called thermography, is not the same as night vision. Night vision operates on the principle of light amplification, so in a totally dark environment light amplification would yield no image where as a thermal imager would based on Emitted Energy + Transmitted Energy + Reflected Energy. Infrared light is emitted or absorbed by molecules when they change their rotational-vibrational movements. It is effective methods to cluster heat generating target. There are numbers of merits of infrared imagery in target tracking such as

$>$ Infrared sensors can detect camouflaged objects.

$>$ Infrared Imaging is not affected by illumination conditions.

$>$ Infrared sensors are passive and involve one-way propagation.

$>$ Infrared imaging has some medical application, essentially in physiotherapy.

$>$ Infrared imaging can successfully penetrate harsh environment such as fog, dust, smoke, snow and rain.

$>$ Infrared imagery can even operate during night time and low light conditions.
$>$ Infrared imagery is capable of catching moving targets in real time.

\subsection{Target Tracking}

Target tracking has been an intensive research area since the early 1960 s, driven primarily by aerospace applications such as radar, sonar, guidance, navigation, and air traffic control. It has also found applications in biological systems, econometrics, robotics and sensor networks. Target is generally described as any area of interest such as persons, mammals, birds, air vehicles, land vehicles, water vehicles, and buildings. Tracking is the process of locating a moving object over time using a camera. The objective of video tracking is to associate target objects in consecutive video frames. To perform video tracking an algorithm analyzes sequential video frames and outputs the movement of targets between the frames so it can be say that Target Tracking can be defined as the problem of estimating the trajectory of an object or target in the image plane as it moves around a scene. There are number of merits of Target Tracking such as

$>$ Tracking is the key for monitoring motion parameters, such as location, velocity, orientation and acceleration, are obtained by targets.

$>$ A target tracking is used for recognizing and understanding target behaviours, especially suffering from illumination, scale, pose variations and occlusion

In this target tracking, first step is infrared video acquisition then split this video into frames. Second step is to do preprocess theses frames means eliminate noise added during video acquisition or transmission. Background modeling or foreground detection in infrared video is used in third step. In this paper background modeling is used such as single frame differencing (SRF) [1], Running Average (RA) and Temporal Median Filter (TMF) [2]. In the fourth step, tracking is performed with Kalman filter and labeling based connected component. There are also some other methods in which tracking is performed before detection, called track before detect (TBD). Here, in this paper tracking is performed after detection of target. In the last step, the performance of tracking algorithm along with selected detection algorithm will be evaluated through performance metrics [3-5] like sensitivity(s) measure, PPV, detection and tracking accuracy. Target tracking can be classified in two forms:

\subsubsection{Single target tracking}

In a single target and single sensor scenario consists of a target whose state evolves through time and is only partially observed 
by a sensor at discrete intervals of time. The objective is to estimate the state of a target given a sequence of observations made by the sensor up to the current time step.

\subsubsection{Multiple target tracking}

In a multiple target tracking scenario, the number of targets changes over time as new targets may appear in the surveillance region due to spontaneous target birth. Moreover, existing targets may not survive to the next time interval and disappear from the scene. The duration for which a target exists in the surveillance region is unknown. At the sensor, not all targets present in its field of view generate measurements.

\section{TYPES OF TRACKING ALOGRITHM}

There are many types of algorithms which usually use in target tracking but in infrared, some other classification of tracking algorithm is used by researcher. Tracking can be done through target representation and localization or by the use of filtering and data association [6]. Target representation and localization is mostly a bottom - up process which has also to cope with change in the appearance of the target. There are some common target representations and localization algorithms such as blob tracking, kernel based tracking, contour tracking. Filtering and data association is mostly a top - down process dealing with the dynamics of the tracked object, learning of scene priors, and evaluation of different hypotheses. Some filters for tracking are such as optimal Bayesian filter, linear filter (Kalman filter), non-linear filter (extended Kalman, unscented Kalman, Gaussian sum filter, particle filter) and techniques for data association are such as nearest neighbour standard filter, probabilistic data association filter, multiple hypothesis tracking, Random sets for multi-target tracking. According to A.Yilmaz et al [7] object tracking is classified into three parts such as point tracking, kernel tracking, silhouette tracking. Point tracking consists of MGE tracker, GOA tracker, Kalman filter, JPDAF, PMHT and kernel tracking consists of mean shift, KLT, Eigntracking, SVM tracker and Silhouette Tracking consists of state space models, heuristic methods, Hough transform, and histogram.

\section{COMPARATIVE ANALYSIS OF DIFFERENT DETECTION ALGORITHM WITH TRACKING ALGORITHM}

In this section, three target detection algorithms such as Single Reference Frame (SRF) [1] and Running Average (RA) [9], Temporal Median Filter [2] along with Kalman filter (KF) is applied on Ohio State University (OSU) Infrared dataset[8]. The detection and tracking performance are analyzed on the parameters[3-5] such as TruePositive, True Negative, false negative, false positive, sensitivity, Positive Predictive Value, Tracking accuracy rate using f-measure, Target TrackingAccuracy rate(TTAR).The description of these parameters are given below

\subsection{True positive (TP)}

It is defined as when some of these frames have the target and the method says they are positive.

$$
\mathrm{TP}=\frac{\sum_{\mathrm{i}} \mathrm{TP}(\mathrm{i})}{\sum_{\mathrm{i}} \mathrm{GT}(\mathrm{i})}
$$

\subsection{False negative (FN)}

It is defined as when some of these frames have the target but the method claims they are no real target.

$$
\mathrm{FN}=\frac{\sum_{\mathrm{i}} \mathrm{FN}(\mathrm{i})}{\sum_{\mathrm{i}} \mathrm{GT}(\mathrm{i})}
$$

\subsection{True negative (TN)}

It is defined as when some of these frames don't have the target and the method also says they are no real target.

$$
\mathrm{TN}=\frac{\sum_{\mathrm{i}} \mathrm{TN}(\mathrm{i})}{\sum_{\mathrm{i}} \mathrm{GT}(\mathrm{i})}
$$

\subsection{False positive (FP)}

It is defined as when some of these frames don't have target but the method claims that target are present.

$$
\mathrm{FP}=\frac{\sum_{\mathrm{i}} \mathrm{FP}(\mathrm{i})}{\sum_{\mathrm{i}} \mathrm{GT}(\mathrm{i})}
$$

\subsection{Sensitivity (S)}

It is defined as the fraction of targets that were correctly detected by the system. High Sensitivity value shows high detection rate but it does not give information of false positives (FP).

$$
\mathrm{S}=\frac{\mathrm{TP}}{\mathrm{TP}+\mathrm{FN}} * 100
$$

\subsection{Positive predictive value (PPV)}

It is defined as the fraction of detections that actually are true targets. High Positive predictive value (PPV) shows a low false alarm rate.

$$
\mathrm{PPV}=\frac{\mathrm{TP}}{\mathrm{TP}+\mathrm{FP}} * 100
$$

\subsection{False alarm rate (FAR)}

It is defined as subtraction ratio of total value and positive predictive value.

$$
\mathrm{FAR}=(1-\mathrm{PPV})
$$

\subsection{Tracking accuracy rate (TAR)}

It is defined as to check the accuracy of tracking method with fmeasure. It is harmonic mean of sensitivity and positive predictive value.

$$
\mathrm{TAR}=1 / \mathrm{n} \sum_{\mathrm{i}=1}^{\mathrm{n}}[\mathrm{x}(\mathrm{i})]^{-1}
$$

Where $\mathrm{x}$ (i) is sensitivity and positive predictive value.

\subsection{Target tracking accuracy rate (TTAR)}

It represents the method's accuracy considering all object configuration errors made by the tracker, false positive, misses, mismatches over all frames.

$$
\text { TTAR }=1-\frac{\sum_{\mathrm{i}}(\mathrm{FN}(\mathrm{i})+\mathrm{FP}(\mathrm{i})+\mathrm{FAR}(\mathrm{i}))}{\sum_{\mathrm{i}} \mathrm{GT}(\mathrm{i})}
$$

In this paper, three different infrared dataset withmultiple targets are used to analyze the detection and tracking accuracy from IEEE OTCBVS WS Series Bench, Roland Miezianko, and Terravic Research Infrared Database[8]. The first thermal data set is two objects enter the FOV from opposite directions and walk toward each other. They stop in the center of the FOV for a while, then turn around and return to their previous positions. The second thermal data set is two objects walk from right to left and briefly stop in the center of the FOV. First objects starts to walk left while second object is stationary. Later, the second object also begins to walk left and third thermal dataset is two objects enter the FOV from opposite directions. They walk 
towards each other and stop in the center of the FOV. Finally, they walk together to the right of the FOV. In which sensor details are Raytheon L-3, Thermal-Eye 2000AS, Format of images $=8$-bit grayscale JPEG, image size $=320 \times 240$ pixels. All the experimented results on these infrared images are shown in Figure1 with Data1(Frameno: 100,203,256,280,375,452), Data2(Frameno:65,125,200,300,390,485),Data3(Frameno:50,13
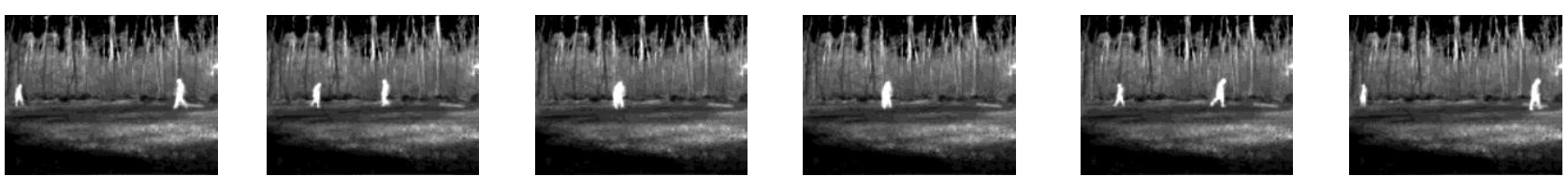

Original OSU Infrared Image Sequence Data 1[8]
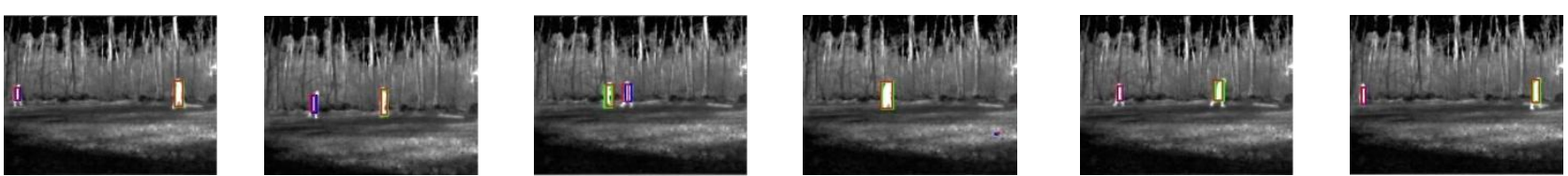

SRF with Kalman Filter Data 1
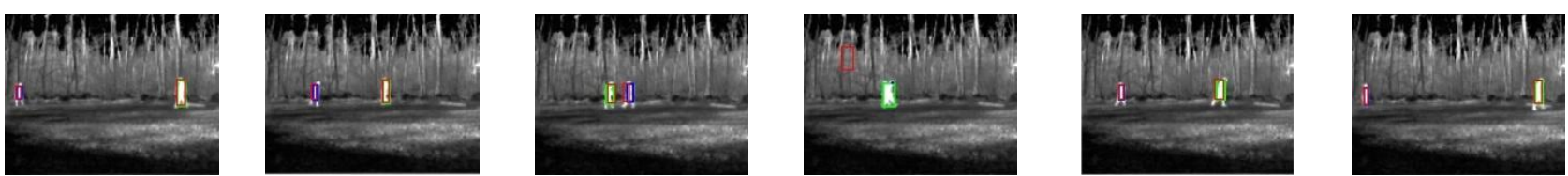

Running Average with Kalman Filter Data 1
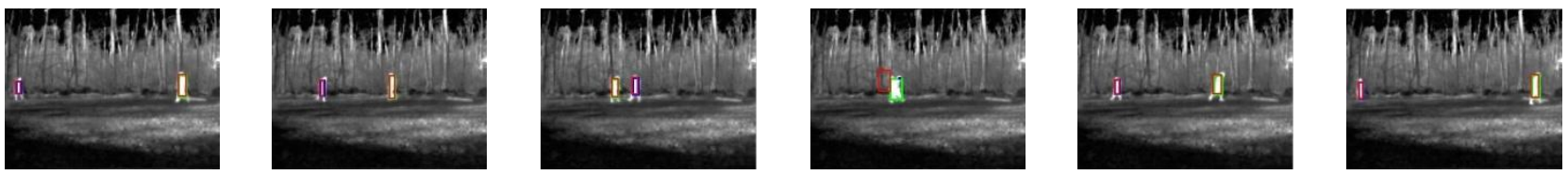

Temporal Median Filter with Kalman Filter Data 1
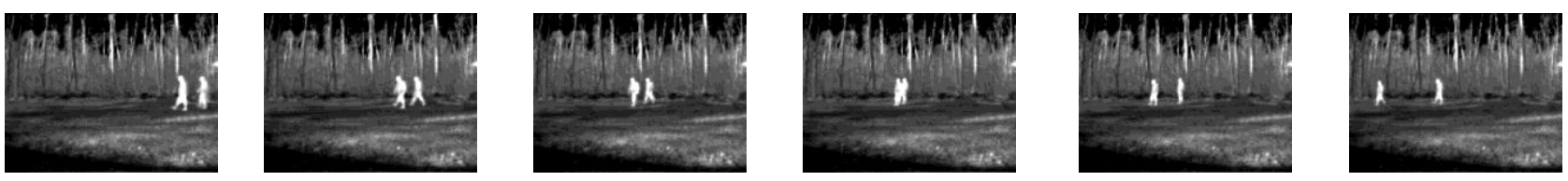

Original OSU Infrared Image Sequence Data 2[8]
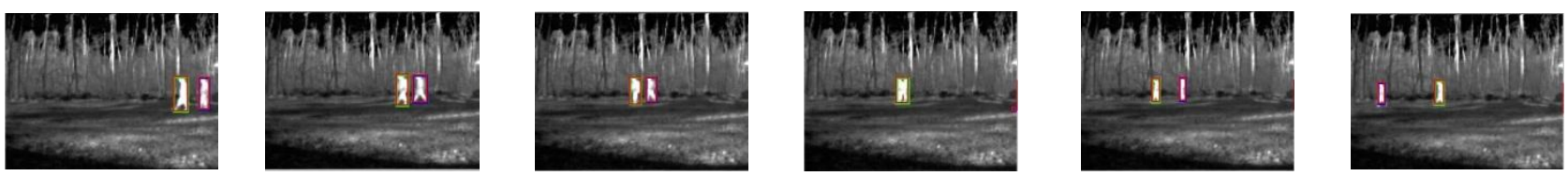

SRF with Kalman Filter Data 2
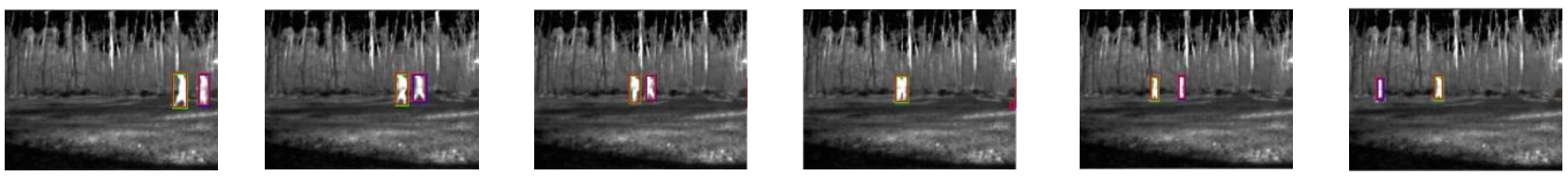

Running Average with Kalman Filter Data 2
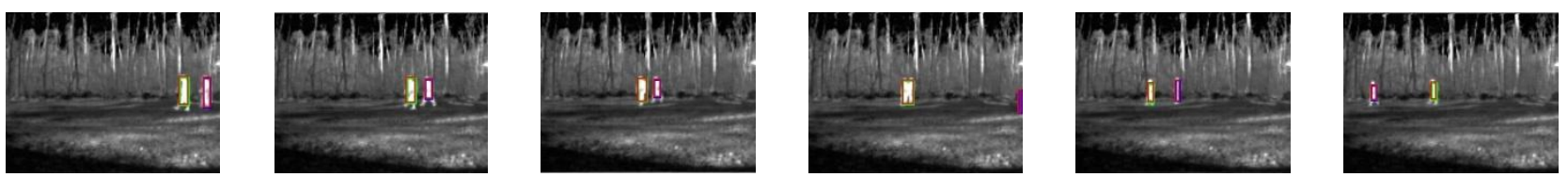

Temporal Median Filter with Kalman Filter Data 2 

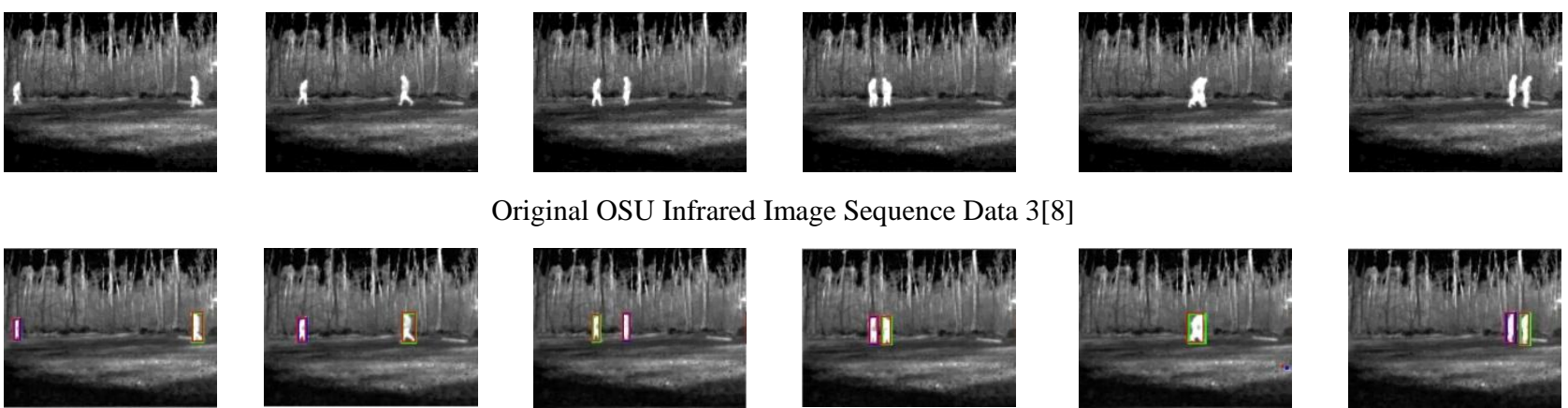

Original OSU Infrared Image Sequence Data 3[8]
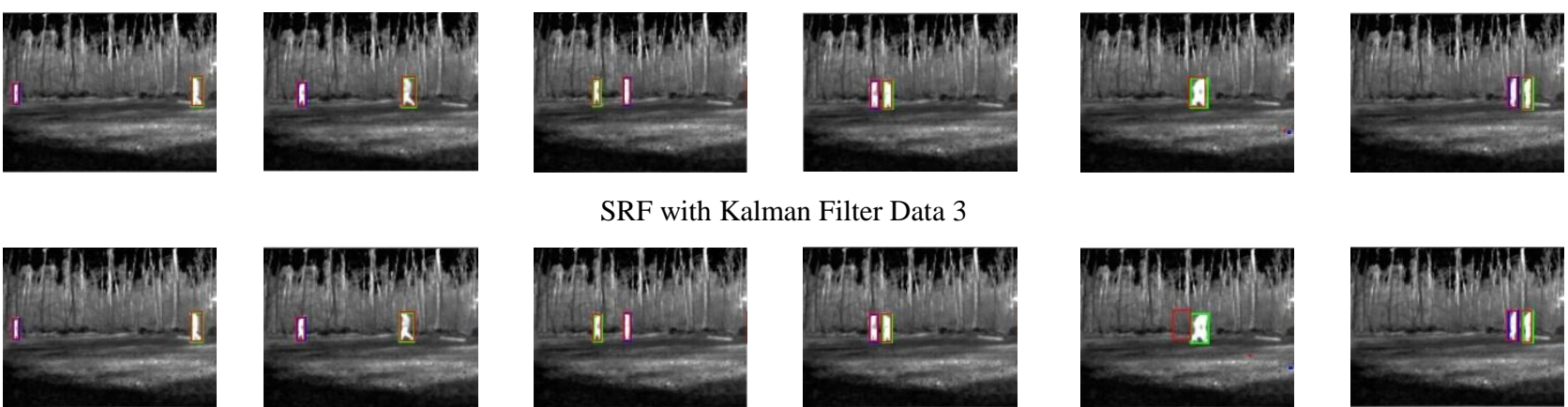

SRF with Kalman Filter Data 3
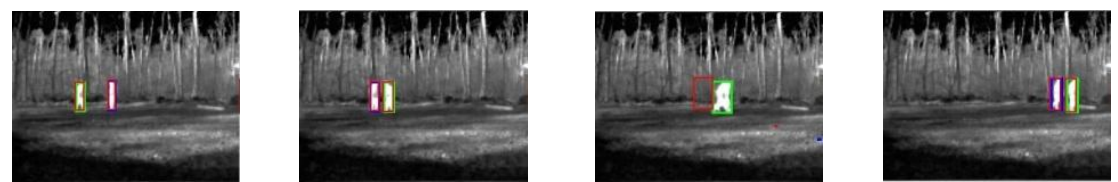

Running Average with Kalman Filter Data 3
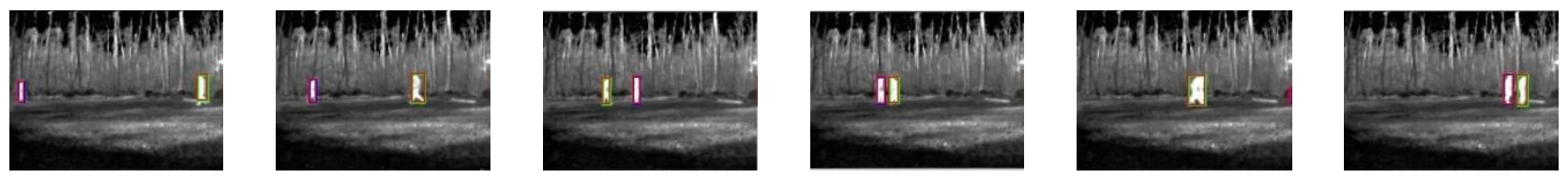

Temporal Median Filter with Kalman Filter Data 3

Figure1. Result of OSU Infrared Image Sequence on SRF, RA and TMF with Kalman Filter

Result analyses of target detection are shown in Table I,Table III, Table V and target tracking are shown in Table II and Table IV, Table VI. To Track the target with single frame differencing [1][10-12] with Kalman filter[13] is not well suitable in infrared imaging. Due to high susceptibility for slight change in atmospheric condition, it is suitable for normal visible images [14]but it cannot track varying intensity object of infrared image sequences. Temporal Median [2] with Kalman[15] is more suitable to track those objects in an environment where there may be slight changes in background or there may be changes in size of the objects but it is not fitted for non-uniform object in infrared imagery. It is also suited for normal visible image or colour image sequences and Running average [9] is one of the popular method for background subtraction and provides an adequate background model which immediately reflects sudden scene change, when RA [9] is used along with Kalman[15] then it provides acceptable accuracy with achieving a high frame rate and having limited memory requirement. It is well suited for small and point targets in normal visible image or colour image or thermal image sequence.

Table I: Result Analysis of Target Detection using Single Reference Frame

\begin{tabular}{|c|c|c|c|c|c|c|c|c|c|c|}
\hline \multirow{2}{*}{ S.No } & \multirow{2}{*}{ Sequence } & \multirow{2}{*}{$\begin{array}{l}\text { Total } \\
\text { Frame }\end{array}$} & \multirow{2}{*}{$\begin{array}{c}\text { Ground } \\
\text { Truth }\end{array}$} & \multicolumn{7}{|c|}{ Single Reference Frame } \\
\hline & & & & TP & FP & FN & $\mathbf{S}$ & PPV & FAR & TAR \\
\hline 1 & $\begin{array}{l}\text { Multiple Person Cross } \\
\text { each other }\end{array}$ & 512 & 485 & 475 & 23 & 1 & 0.997 & 0.953 & 0.047 & 0.974 \\
\hline 2 & $\begin{array}{l}\text { Multiple Person } \\
\text { comes and move the } \\
\text { same Direction }\end{array}$ & 696 & 680 & 678 & 2 & 0 & 1 & 0.997 & 0.003 & 0.998 \\
\hline 3 & $\begin{array}{c}\text { Multiple Person } \\
\text { comes from different } \\
\text { direction and move in } \\
\text { same direction }\end{array}$ & 720 & 690 & 546 & 1 & 1 & 0.998 & 0.998 & 0.002 & 0.998 \\
\hline
\end{tabular}


Table II: Result Analysis of Target Tracking using SRF with Kalman

\begin{tabular}{|c|c|c|c|c|c|c|c|c|c|c|c|}
\hline \multirow{2}{*}{ S.No } & \multirow{2}{*}{ Sequence } & \multirow{2}{*}{$\begin{array}{c}\text { Total } \\
\text { Frame }\end{array}$} & \multirow{2}{*}{$\begin{array}{c}\text { Ground } \\
\text { Truth }\end{array}$} & \multicolumn{8}{|c|}{ Single Reference Frame with Kalman } \\
\hline & & & & TP & FP & FN & $\mathbf{S}$ & PPV & FAR & TAR & TTAR \\
\hline 1 & $\begin{array}{l}\text { Multiple Person Cross } \\
\text { each other }\end{array}$ & 512 & 485 & 430 & 20 & 34 & 0.926 & 0.955 & 0.045 & 0.940 & 0.888 \\
\hline 2 & $\begin{array}{l}\text { Multiple Person } \\
\text { comes and move the } \\
\text { same Direction }\end{array}$ & 696 & 680 & 672 & 1 & 26 & 0.962 & 0.998 & 0.002 & 0.979 & 0.960 \\
\hline 3 & $\begin{array}{c}\text { Multiple Person } \\
\text { comes from different } \\
\text { direction and move in } \\
\text { same direction }\end{array}$ & 720 & 690 & 506 & 1 & 38 & 0.930 & 0.998 & 0.002 & 0.962 & 0.943 \\
\hline
\end{tabular}

Table III: Result Analysis of Target Detection using (Moving)Running Average

\begin{tabular}{|c|c|c|c|c|c|c|c|c|c|c|}
\hline \multirow{2}{*}{ S.No } & Sequence & Total & \multirow{2}{*}{$\begin{array}{c}\text { Ground } \\
\text { Frame }\end{array}$} & Truth & \multicolumn{6}{|c|}{ (Moving)Running Average } \\
\cline { 6 - 10 } & & & TP & FP & FN & S & PPV & FAR & TAR \\
\hline 1 & $\begin{array}{c}\text { Multiple Person Cross } \\
\text { each other }\end{array}$ & 512 & 485 & 441 & 23 & 1 & 0.997 & 0.953 & 0.047 & 0.974 \\
\hline 3 & $\begin{array}{c}\text { Multiple Person comes } \\
\text { and move the same } \\
\text { Direction }\end{array}$ & 696 & 680 & 670 & 2 & 0 & 1 & 0.997 & 0.003 & 0.998 \\
\hline & $\begin{array}{c}\text { Multiple Person comes } \\
\text { from different } \\
\text { direction and move in } \\
\text { same direction }\end{array}$ & 720 & 690 & 566 & 1 & 1 & 0.998 & 0.998 & 0.002 & 0.998 \\
\hline
\end{tabular}

Table IV: Result Analysis of Target Tracking using RAwith Kalman

\begin{tabular}{|c|c|c|c|c|c|c|c|c|c|c|c|}
\hline \multirow{2}{*}{ S.No } & \multirow{2}{*}{ Sequence } & \multirow{2}{*}{$\begin{array}{l}\text { Total } \\
\text { Frame }\end{array}$} & \multirow{2}{*}{$\begin{array}{c}\text { Ground } \\
\text { Truth }\end{array}$} & \multicolumn{8}{|c|}{ (Moving) Running Average with Kalman } \\
\hline & & & & TP & FP & FN & $\mathbf{S}$ & PPV & FAR & TAR & TTAR \\
\hline 1 & $\begin{array}{l}\text { Multiple Person } \\
\text { Cross each other }\end{array}$ & 512 & 485 & 400 & 1 & 26 & 0.947 & 0.997 & 0.003 & 0.971 & 0.9443 \\
\hline 2 & $\begin{array}{l}\text { Multiple Person } \\
\text { comes and move the } \\
\text { same Direction }\end{array}$ & 696 & 680 & 665 & 4 & 51 & 0.967 & 0.994 & 0.006 & 0.980 & 0.9235 \\
\hline 3 & $\begin{array}{l}\text { Multiple Person } \\
\text { comes from different } \\
\text { direction and move } \\
\text { in same direction }\end{array}$ & 720 & 690 & 523 & 2 & 20 & 0.917 & 0.996 & 0.004 & 0.954 & 0.9652 \\
\hline
\end{tabular}

Table V: Result Analysis of Target Detection using Temporal Median Filter

\begin{tabular}{|c|c|c|c|c|c|c|c|c|c|c|}
\hline \multirow{2}{*}{ S.No } & \multirow{2}{*}{ Sequence } & \multirow{2}{*}{$\begin{array}{c}\text { Total } \\
\text { Frame }\end{array}$} & \multirow{2}{*}{$\begin{array}{c}\text { Ground } \\
\text { Truth }\end{array}$} & \multicolumn{7}{|c|}{ Temporal Median Filter } \\
\hline & & & & TP & FP & $\mathbf{F N}$ & $\mathbf{S}$ & PPV & FAR & TAR \\
\hline 1 & $\begin{array}{c}\text { Multiple Person Cross } \\
\text { each other }\end{array}$ & 512 & 485 & 445 & 0 & 7 & 0.984 & 1 & 0 & 0.992 \\
\hline 2 & $\begin{array}{l}\text { Multiple Person } \\
\text { comes and move the } \\
\text { same Direction }\end{array}$ & 696 & 680 & 595 & 1 & 22 & 0.964 & 0.998 & 0.002 & 0.980 \\
\hline 3 & $\begin{array}{c}\text { Multiple Person } \\
\text { comes from different } \\
\text { direction and move in } \\
\text { same direction }\end{array}$ & 720 & 690 & 670 & 0 & 6 & 0.991 & 1 & 0 & 0.995 \\
\hline
\end{tabular}


Table VI: Result Analysis of Target Tracking using TMF with Kalman

\begin{tabular}{|c|c|c|c|c|c|c|c|c|c|c|c|}
\hline \multirow{2}{*}{ S. No } & \multirow{2}{*}{ Sequence } & \multirow{2}{*}{$\begin{array}{l}\text { Total } \\
\text { Frame }\end{array}$} & \multirow{2}{*}{$\begin{array}{c}\text { Ground } \\
\text { Truth }\end{array}$} & \multicolumn{8}{|c|}{ Temporal Median Filter with Kalman } \\
\hline & & & & $\mathbf{T P}$ & FP & $\mathbf{F N}$ & $\mathbf{S}$ & PPV & FAR & TAR & TTAR \\
\hline 1 & $\begin{array}{l}\text { Multiple Person } \\
\text { Cross each other }\end{array}$ & 512 & 485 & 420 & 1 & 22 & 0.941 & 0.997 & 0.003 & 0.968 & 0.952 \\
\hline 2 & $\begin{array}{l}\text { Multiple Person } \\
\text { comes and move the } \\
\text { same Direction }\end{array}$ & 696 & 680 & 547 & 1 & 22 & 0.914 & 0.998 & 0.002 & 0.954 & 0.961 \\
\hline 3 & $\begin{array}{l}\text { Multiple Person } \\
\text { comes from different } \\
\text { direction and move } \\
\text { in same direction }\end{array}$ & 720 & 690 & 670 & 4 & 47 & 0.971 & 0.994 & 0.006 & 0.982 & 0.928 \\
\hline
\end{tabular}

In this paper, the implementation of the algorithm on benchmark data set of Ohio state university infrared image sequence [8] are shown and done some performance test like fmeasure for sensitivity and target accuracy rate and target tracking accuracy rate are calculated that are shown in different

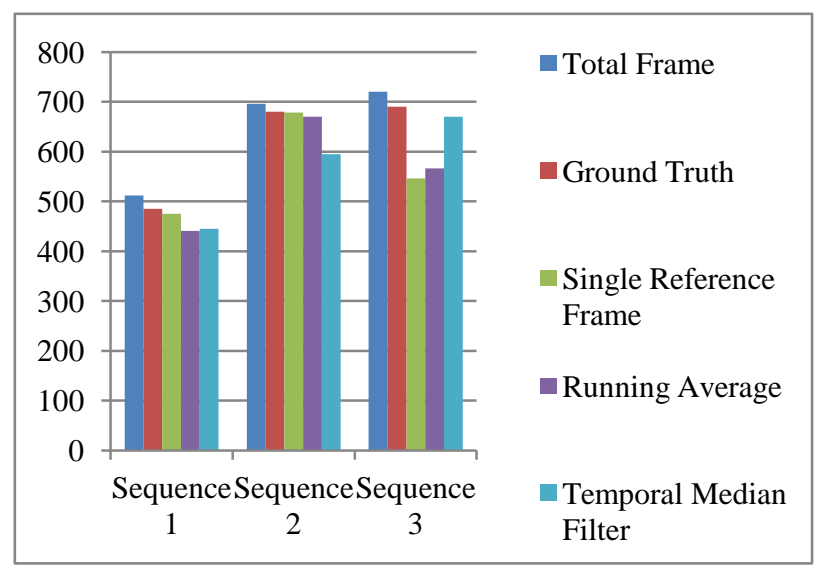

Performance Analysis based on True Positive (TP) of Detection Algorithm

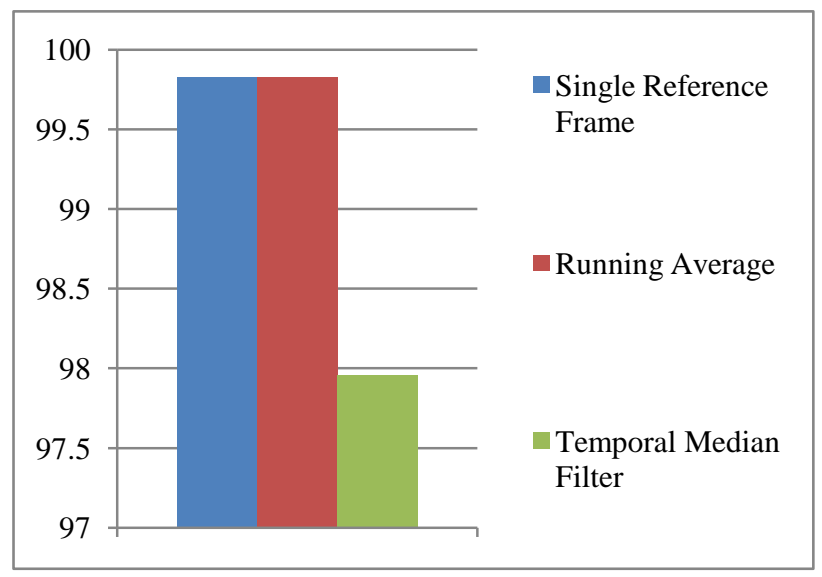

Performance Analysis based on Sensitivity (S) of Detection Algorithm graph. With the help of this statistical data it can be analyze the detection and tracking accuracy of the multiple targets with SRF [1] with Kalman, RA [9] with Kalman and TMF [2] with Kalman. It analyzes multiple targets in SRF [1] and RA [9],TMF [2] with Kalman with graph as shown in Figure2.

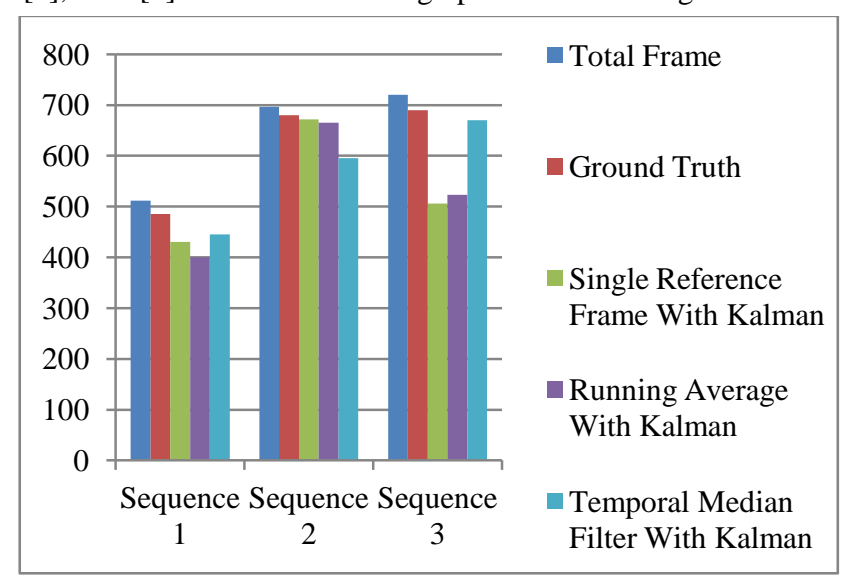

Performance Analysis based on True Positive (TP) of Tracking Algorithm

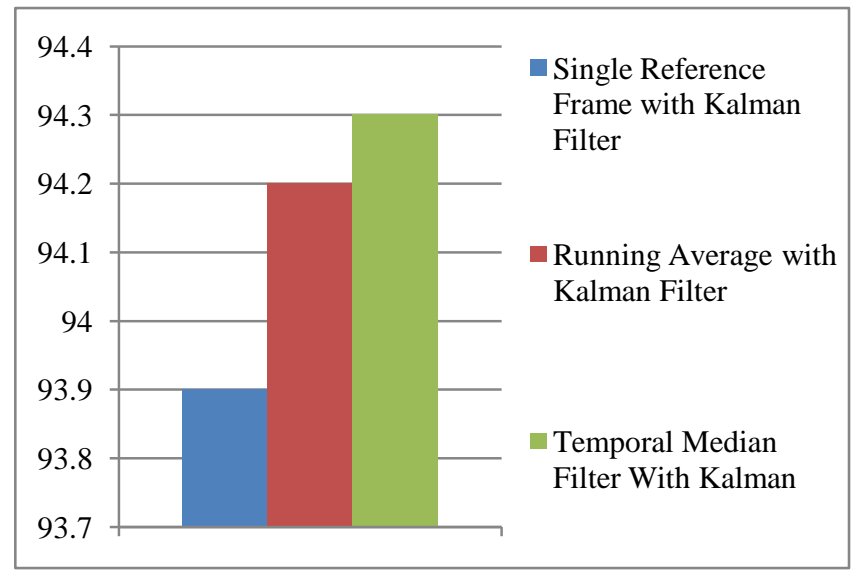

Performance Analysis based on Sensitivity (S) of Tracking Algorithm 


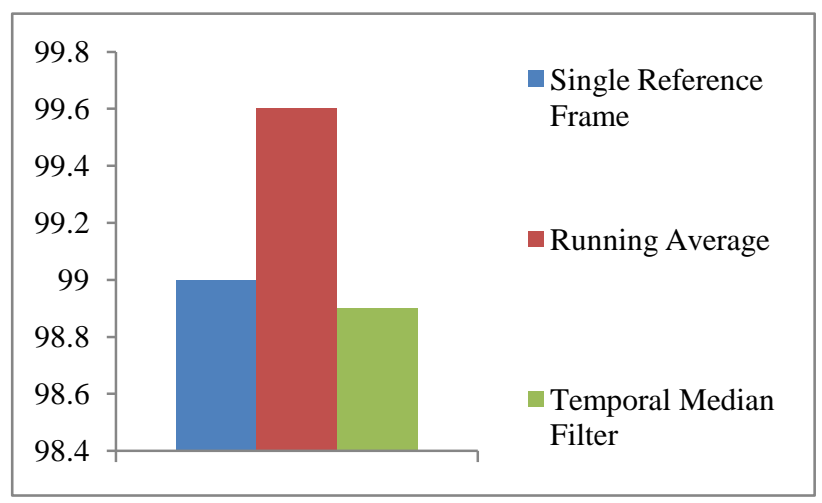

Performance Analysis based on f-measure of Detection Algorithm

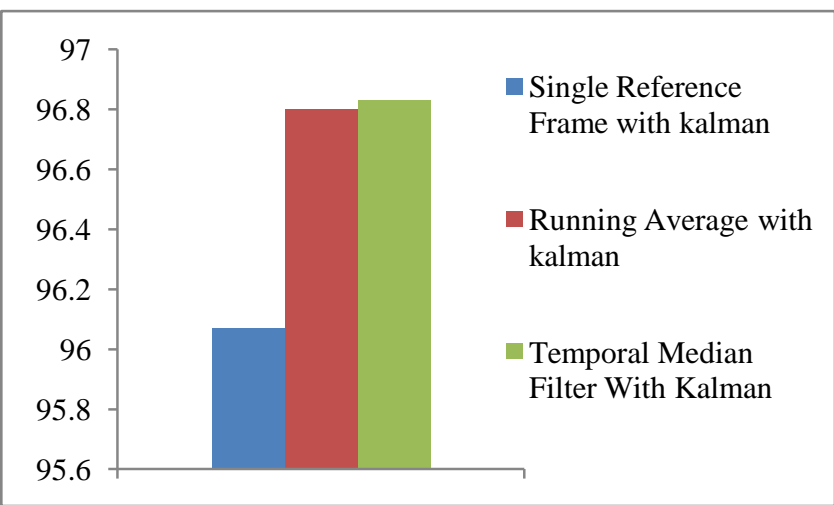

Performance Analysis based on f-measure of Tracking Algorithm

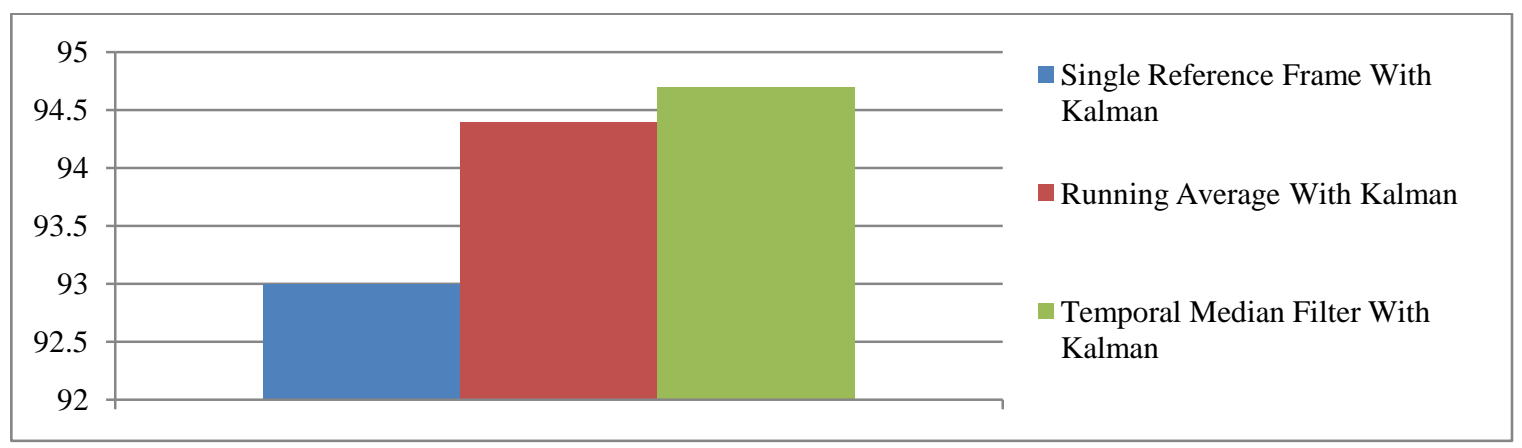

Performance Analysis based on Accuracy of Tracking Algorithm

Figure 2. Performance Parameters Analysis of target tracking with Kalman Filter

\section{CONCLUSION}

In this paper, a brief review of different approaches of multiple targets tracking [16-18] in infrared imaging is presented. Intruder detection and tracking system are essential parts of security in every field such as border security, sea shore security, traffic monitoring and robotics based rescue operations. There are many obstacles such as noise, directional view, pose, illumination that affects the overall performance of the tracking. Temporal median filter [19-22] (TMF) with kalman filter (KF) performs better in the case of multiple targets tracking in thermal imagery. TMF with $\mathrm{KF}$ is more sensitive towards target detection and tracking and its positive predictive value is also high in comparison of other methods. Experimental results of SRF, RA and TMF with Kalman Filter on OSU dataset with multiple targets are analyzed and computed.

\section{ACKNOWLEDGMENT}

The authors would like to thank to IEEE OTCBVS WS Series Bench, Roland Miezianko, and Terravic Research Infrared Database.

\section{REFERENCES}

[1] Chaohui, Z., "An improved moving object detection algorithm based on frame difference and edge detection." IEEE Fourth International Conference on Image and Graphics, 2007.

[2] Mao-Hsiung H., Jeng-Shyang P. and Chaur-Heh H., "Speed Up Temporal Median Filter for Background Subtraction," First International Conference on Pervasive Computing Signal Processing and Applications (PCSPA), pp.297,300, Sept. 2010.
[3] Bashir, F. and Fatih P., "Performance evaluation of object detection and tracking systems." In PETS 6, 2006.

[4] Nascimento, J. and Jorge S. M., "New performance evaluation metrics for object detection algorithms." Proceedings of IEEE PETS Workshop. 2004.

[5] Mariano and Vladimir Y., et al. "Performance evaluation of object detection algorithms." IEEE 16th International Conference on Pattern Recognition, Vol. 3, 2002.

[6] Cmaniciu, R. and Meer, "Kernel-based object tracking," IEEE Transactions on Pattern Analysis and Machine Intelligence, vol.25, no.5, pp.564-577, May 2003.

[7] Yilmaz, A., Omar J., and Mubarak S. "Object tracking: A survey."Acm Computing Surveys (CSUR), 2006.

[8] http://www.cse.ohio-state.edu/otcbvs-bench (accessed on 20 Jan 2013).

[9] Elhabian, S., Khaled, S., and Sumaya "Moving object detection in spatial domain using background removal techniques-state-of-art." Recent patents on computer science, 2008

[10] Majid, R., Jamali, G., Frizado, B., "Avian detection \& tracking algorithm using infrared imaging," IEEE International Conference on Electro/Information Technology (EIT), pp.1-4, 2012.

[11] McIvor, "Background Subtraction Techniques," Proc. of Image and Vision Computing, 2000. 
[12] Piccardi, "Background subtraction techniques: a review," in Proc. IEEE International Conference Systems, Man, Cybernetics, pp. 3099-3104, 2004.

[13] Welch, G. and Gary B., "An introduction to the Kalman filter." 1995.

[14] Nummiaro, K., Esther K.-M.and Luc V. G., "An adaptive color-based particle filters." Image and Vision Computing, 2003.

[15] Chen, S. Y. "Kalman filter for robot vision: a survey." IEEE Transactions on Industrial Electronics 2012

[16] Bernardin, K., Alexander E. and Rainer S., "Multiple object tracking performance metrics and evaluation in a smart room environment." Sixth IEEE International Workshop on Visual Surveillance, in conjunction with ECCV. Vol. 90. 2006.

[17] Ristic, B., Sanjeev A. and Neil G., "Beyond the Kalman filter: Particle filters for tracking applications." Artech House Publishers, 2004.

[18] Lee, S. J., et al. "Human tracking with an infrared camera using a curve matching framework." EURASIP Journal on Advances in Signal Processing 2012.

[19] Deshpande andSuyog D., et al. "Max-mean and maxmedian filters for detection of small targets." SPIE's International Symposium on Optical Science, Engineering, and Instrumentation. International Society for Optics and Photonics, 1999.

[20] Loveridge and Jennifer C. "Adaptive, hybrid median filter for temporal noise suppression." U.S. Patent No. 5,Jan. 1995

[21] Li, R., Bing Z., and Ming L. L., "Reliable motion detection/compensation for interlaced sequences and its applications to deinterlacing." IEEE Transactions on Circuits and Systems for Video Technology, 2000.

[22] Silverman, J., et al. "Temporal filtering for point target detection in staring IR imagery: II. Recursive variance filters." Aerospace/Defense Sensing and Controls. International Society for Optics and Photonics, 1998.

[23] Lai, K., et al. "A large-scale hierarchical multi-view rgb-d object dataset." IEEE International Conference on Robotics and Automation (ICRA), 2011

[24] Huang, S.-C., "An advanced motion detection algorithm with video quality analysis for video surveillance systems." IEEE Transactions on Circuits and Systems for Video Technology, 2011.

[25] Bae, T.-W., "Small target detection using bilateral filter and temporal cross product in infrared images." Infrared Physics \& Technology, 2011.

[26] Kim, T., Lee and Paik, "Combined shape and featurebased video analysis and its application to non-rigid object tracking." IET Image Processing, 2011.

[27] Genin, L., et al. "Point object detection using a NL-means type filter." IEEE International Conference on Image Processing (ICIP), 2011.

[28] Neri, A., et al. "Automatic moving object and background separation." Signal Processing, 1998

[29] Zheng Yi and Fan L., "Moving object detection based on running average background and temporal difference," International Conference on Intelligent Systems and Knowledge Engineering (ISKE), pp.270, 272, Nov. 2010.

[30] Sheikh, Y., and Mubarak S., "Bayesian object detection in dynamic scenes." IEEE Computer Society Conference on Computer Vision and Pattern Recognition, 2005 